\title{
Experimental and Numerical Investigation of Thermal Chaotic Mixing in a T-shaped Microchannel
}

\section{Introduction}

Microfluidic systems have been attracting great attentions over past decades. Applications based on microfluidic systems have been emerging in many fields such as biology and chemistry area. In these fields, analysis such as DNA hybridization, drug delivery and chemical reactions often require rapid mixing [1]. However, due to the dominating laminar flow on the microscale, mixing in the microfluidic system relies mainly on molecular diffusion, which resulting low mixing efficiency [2]. Due to this particular limitation, more and more researchers have been focusing on micromixers to enhance mixing.

Micromixers based on different working principles have been proposed and investigated systematically. Generally speaking, micromixers can be categorized as passive micromixers and active micromixers [2]. Passive micromixers rely on diffusion and chaotic advection to enhance mixing without any external disturbance, while active micromixers utilize external disturbance to enhance mixing. Chaotic mixing is recognized as an efficient way to enhance mixing [3]. Chaotic advection can increase the contact surface area and decrease the diffusion path, and the mixing can be enhanced. For passive micromixers, chaotic advection can be induced by adding special geometry in the mixing channel. For active micromixers, chaotic advection 
can be generated by external disturbance.

Coupling of chaotic mixing with heat transfer has been attracting more and more attention from the research community. The motivation of this study is due to the significant difference between mass transfer rate and heat transfer rate [4]. The thermal diffusivity is three orders higher than the mass diffusivity. Taking heat transfer into consideration will have significant impact on the advection-diffusion problem [5, 6]. Mokrani et al. [7] studied the effect of chaotic advection on heat transfer at low Reynolds number. The chaotic heat exchanger was found to be more effective than the helical heat exchanger. Chagny et al. [8] investigated chaotic heat transfer characteristics of heat exchanger. Chaotic heat transfer was found to be efficient at low Reynolds number. Acharya et al. [9] analyzed the phenomena of steady heat transfer enhancement due to the chaotic advection. Lemenand et al. [10] implemented a thermal model to simulate heat transfer characteristic in a twisted pipe with chaotic configuration. Lefevre et al. [11] studied chaotic advection and heat transfer enhancement in stokes flow experimentally. The heat transfer rate between solid boundary and the fluid was found to be enhanced in the chaotic configuration. Kumar et al. [12] investigated the laminar convective heat transfer in chaotic configuration numerically. Chaotic mixing was found to enhance the heat transfer about 25-36\%. Yamagishi et al. [13] numerically studied mixing performance and heat transfer in multiple pipe bends for different switching angles. Lester et al. [14] investigated a Rotated Arc Mixer (RAM) theoretically. The mixing consists of an 
inner stationary cylinder which was wrapped by a concentric outer rotating cylinder. The heat and mass transfer can be enhanced in this geometry.

Based on discussions above, we can see that regarding to the coupling of chaotic mixing with heat transfer, researchers focus on the heat transfer enhancement which is due to chaotic advection. Little literature is about the mass mixing enhancement which is due to heat transfer in chaotic configuration. The main objective of this paper is to study the effect of heat transfer on mixing performance in a chaotic micromixer with simple geometry experimentally and numerically. The micromixer consists of a heating cylinder embedded in a T-shaped microchannel. Laser Induced Fluorescence (LIF) method is employed to measure the concentration and temperature fields in the microchannel. Experiments were carried out to show the feasibility of utilizing heat transfer to enhance mixing performance in a chaotic configuration. The numerical simulation is employed to provide a better understanding of the enhanced mixing performance which is due to heat transfer.

\section{Experiment}

\subsection{Fluorescence imaging setup}

The schematic illustration of the fluorescence imaging setup is shown in Fig. 1, consisting of four main components: an illumination system, an optical system, a coupled charge device (CCD) camera and a personal computer (PC) based control 
system. A mercury lamp with the wave length of $540 \mathrm{~nm}$ is employed as the illumination source for the fluorescence imaging setup.

The Nikon inverted microscope (Model ECLIPSE TE2000-S) with a set of epifluorescent attachments is used as the optical system. Excitation filter, dichroic mirror and emission filter constitute the filter cube. Emission filters are used in the measurements to select the specific emission wavelength of the sample and to remove traces of excitation light.

An interline transfer CCD camera (Sony ICX 084) is used for recording the images. The resolution of the camera is $1324 \times 1024$ pixels with 16 bits grayscale. The active area of the CCD sensor is $5 \mathrm{~mm} \times 3 \mathrm{~mm}$. The exposure time for recording the image is $7 \times 10^{4} \mu s$.

\subsection{Experiment on thermal chaotic mixing}

The T-shaped microfluidic device used in the experiment is shown in Fig. 2(a), while the schematic illustration of focused area captured by the CCD camera is shown in Fig. 2(c). The fabrication is based on the lamination thermal bonding technique [15]. In this method, three polymethylmethacrylate (PMMA) plates $(50 \mathrm{~mm} \times 25 \mathrm{~mm})$ were bonded together to form a closed microfluidic channel with inlet and outlet holes. Thus, the middle PMMA defines the depth of the channel. A copper cylinder was inserted into the microchannel from the hole of the top PMMA plate. The length and diameter of the cylinder was $10 \mathrm{~mm}$ and $0.67 \mathrm{~mm}$ respectively. An electric resistance wire was wrapped on the copper cylinder circumference. In this experiment, the 
vertical portion of the T-shaped microchannel has a cross section of $1000 \mu \mathrm{m} \times 2000 \mu \mathrm{m}$ and a length of $3 \mathrm{~cm}$, while the straight portion of the T-shaped microchannel has a cross section of $1000 \mu \mathrm{m} \times 2000 \mu \mathrm{m}$ and a length of $3 \mathrm{~cm}$.

DI-water with fluorescent dye (fluorescein disodium salt $\mathrm{C}_{20} \mathrm{H}_{10} \mathrm{Na}_{2} \mathrm{O}_{5}$, Acid Yellow 73) and DI-water was introduced into inlet port A and inlet port B respectively by two identical syringes, which were driven by a syringe pump (Cole-Parmer, 74900$05,0.2-500 \mathrm{ml} / \mathrm{h}$, accuracy of $0.5 \%$ ) to supply the same volumetric flow rates. A low voltage power supply (GW Model GPC- 30300) was used to supply voltage to the electric resistance wire to enable heating of the copper cylinder. The images of the mixing process around the heater for different flow rates were captured by the CCD camera. After capturing the images, the mixing process of the two fluids were determined by a customized MATLAB program. This program removes the noise of the collected images, and then calculates concentration distribution in the microchannel. A linear relationship is assumed between the fluorescence intensity and the concentration of the fluorescent dye [16].

To quantify mixing efficiency of a micromixer, a region of interest (ROI) needed to be defined in the captured images. After that, the average intensity value of each row $i$ in the ROI was calculated as $I_{i}$. This value was then normalized by the maximum and minimum fluorescence intensity value:

$$
I_{i}^{*}=\frac{I_{i}-I_{\min }}{I_{\max }-I_{\min }}
$$

Where $I_{i}^{*}$ is the normalized average intensity value of each row $i . I_{\max }$ and $I_{\min }$ are the maximum and minimum fluorescence intensity value in the unmixed states. 
The mixing efficiency is defined as:

$$
\eta=1-\frac{\sqrt{\frac{1}{N} \sum_{i=1}^{N}\left(I_{i}^{*}-I_{\infty}^{*}\right)^{2}}}{\sqrt{\frac{1}{N} \sum_{i=1}^{N}\left(I_{0 i}^{*}-I_{\infty}^{*}\right)^{2}}}
$$

where $N$ is the number of rows in the ROI, $I_{0 i}^{*}$ is the normalized average intensity value of each row $i$ without mixing taking place (e.g., value 0 or 1 in this study),

$I_{\infty}^{*}$ is the normalized average intensity value of each row $i$ with complete mixing taking place (e.g., value 0.5 in this study).

To quantify heating effect of the copper cylinder, DI-water and DI-water with fluorescent dye (Rhodamine B) were introduced into inlet A and inlet B respectively by two identical syringe pumps, which were driven by a syringe pump (Cole-Parmer, $74900-05,0.2-500 \mathrm{ml} / \mathrm{h}$, accuracy of $0.5 \%$ ). A proportional relationship is assumed between fluorescence intensity and temperature [17]. The resulting intensity (normalized by the intensity at $25^{\circ} \mathrm{C}$ ) versus temperature is shown in Fig. 3 .

\section{Two-dimensional numerical simulation}

The schematic of the computational domain is shown in Fig. 2(b). The gray color parts are the PMMA plates. The heater is placed in the T-shaped microchannel. The heat and mass transfer process of a thermal chaotic mixing is a conjugate forced convection-conduction problem. Therefore, it is needed to take into consideration of the heat conduction in the PMMA plates and convective heat and mass transfer in the fluid. Furthermore, the concentration and temperature fields obtained from the numerical simulation can be used to confirm the trend of experimental results. 
The heat and mass transfer process of thermal chaotic mixing occurs in two regions including the solid region (PMMA plates) and the fluid region (fluid flow). The temperature distribution in the solid region is governed by the two-dimensional heat conduction equation while the temperature and concentration distribution in the fluid region is governed by the two-dimensional heat and mass conduction-convection equation. In the numerical simulation model, laminar, incompressible, Newtonian fluid and steady flow are assumed. The density, specific heat, thermal conductivity and viscosity of DI-water are allowed to vary with temperature[18].

\subsection{Governing equations}

The governing equations for the fluid flow and heat and mass transfer characteristics of thermal chaotic mixing can be written in a Cartesian coordinate system as follow.

\subsubsection{Fluid velocity field}

With the assumption of the incompressible Newtonian fluid, the fluid velocity field is described by the continuity and Navier-Stokes equations.

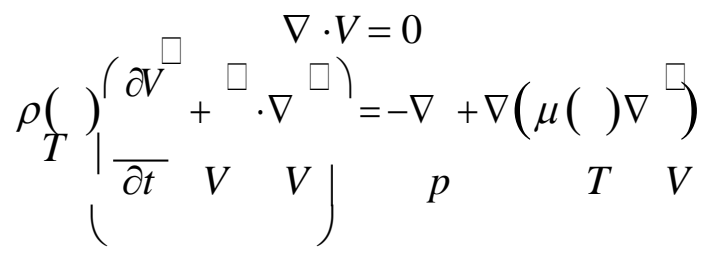

Where $V$ is the fluid velocity vector, $\rho$ and $p$ are the fluid density and hydrodynamic pressure respectively, $\rho(T)$ and $\mu(T)$ are the fluid density and viscosity respectively, whose dependence on temperature are given as follows, 


$$
\begin{gathered}
\rho=\frac{999.8+18.2 \times T-7.9 \times 10^{-3} \times T^{2}-5.5 \times 10^{-5} \times T^{3}+1.5 \times 10^{-7} \times T^{4}-3.9 \times 10^{-10} T^{5}}{1+1.8 \times 10^{-2} \times T} \\
\mu=2.414 \times 10^{-5} \times 10^{247.8 /(T-140)}
\end{gathered}
$$

\subsubsection{Fluid and channel wall temperaturefields}

The temperature field in the microchannel is governed by the energy equation expressed as

$$
\rho(T) c_{p}(T)\left(\frac{\partial T}{\partial t}+V \cdot \nabla T\right)=\nabla(k(T) \nabla T)
$$

where $c_{p}$ and $k$ are specific heat and the thermal conductivity of the working fluid respectively, whose dependence on temperature are given as follows,

$$
\begin{gathered}
C_{p}=8958.9-40.535 \times T+0.11243 \times T^{\stackrel{2}{-}} 1.0138 \times 10^{\stackrel{-4}{\times}} T^{3} \\
k=-0.58166+6.3555 \times 10^{-3} \times T-7.9643 \times 10^{-6} \times T^{2}
\end{gathered}
$$

In addition, since the thermal boundary condition on the channel wall is unknown, a conjugate heat transfer problem has to be solved by simultaneously considering the heat conduction in the channel wall. The temperature field in the channel wall is governed by the heat conduction equation expressed as

$$
\rho_{s} \frac{\partial T}{\partial t}=k_{s} \nabla^{2} T
$$

where $\rho_{s}, c_{p s}, k_{s}$ are the specific heat, density, and thermal conductivity of the microchannel wall (PMMA plate), respectively.

\subsubsection{Fluid Concentration field}


The temperature field in the microchannel is governed by the mass transport equation expressed as:

$$
\frac{\partial C}{\partial t}+V \cdot \nabla C=\nabla(D(T) \nabla C)
$$

where $C$ is the concentration of fluorescent dye, and $D(T)$ is the temperature dependent mass diffusion coefficient of fluorescent dye, given by $D(T)=D_{0}\left[1+0.02\left(T-T_{0}\right)\right] \quad[19] . D_{0}$ is the mass diffusion coefficient of the fluorescent dye at room temperature $T_{0}$.

\subsection{Initial and boundary conditions}

The temperature, concentration and transverse velocity along the y direction at the inlet are specified. The temperature, concentration and velocity at the inlet are assumed to be uniform. The non-slip and zero mass flux boundary conditions are used for the walls. Constant temperature boundary condition is set for the left and right wall. Nature convection boundary condition is set for the top and bottom wall. The heat transfer coefficient of natural convection is chosen to be $15 \mathrm{Wm}^{-2} \mathrm{~K}^{-1}$. At the outlet, zero gradient conditions are imposed on the transverse velocity, temperature and concentration.

\subsection{Numerical method}

Since the governing equations for the velocity, temperature and concentration fields are strongly coupled in the temperature dependent parameters. The numerical method is inherently used to solve this coupled problem. COMSOL Multiphysics ${ }^{\mathrm{TM}}$ 
was employed to numerically solve governing equations (3) - (11) to obtain the fluid velocity field, and the temperature field. A grid independence test was conducted to ensure the accuracy of the numerical simulation results.

\section{Results and discussion}

The parameters investigated in the experiments and numerical simulations are the electric voltage applied to the copper cylinder and the flow rates. For different operating conditions, the measurement and numerical simulation were taken under steady-state condition.

\subsection{Effect of power input}

The typical images taken in the experiments under the effect of power input and corresponding heating temperature are shown in Fig. 4, where the flow rate is kept at $1 \mathrm{ml} / \mathrm{h}$. When no voltage was applied on the resistance wire, the mixing behavior is simply a chaotic mixing. Since the copper cylinder is put in the middle of the microchannel, the concentration distribution at the around of the cylinder is almost symmetry. There was almost no occurrence of mixing at the surrounding of the cylinder, as suggested by a clear interface between the two fluids in the microchannel shown in Fig. 4(a). This is expected in the case of low Reynolds number in the microchannel, mixing is dominated by molecular diffusion. However, once a voltage was applied on the resistance wire, an enhancement in the mixing could be observed in Fig. 4(b)-(d), which depicted the effect of power input on the mixing behavior. 
When a $1 \mathrm{v}$ voltage was applied on the resistance wire, the copper cylinder will be inherently heated. Thus, the copper cylinder becomes a heater. The interface between the two fluids in the present microfluidic structure becomes obscure, suggesting occurrence of mixing between two fluids as shown in Fig. 4(b). But the mixing was still poor (indicated by the large dark area in the ROI). However, mixing enhancement can be observed when a $1.5 \mathrm{v}$ and $2 \mathrm{v}$ voltage was applied on the resistance wire respectively as shown in Fig. 4(c) and (d). This could be due to that the higher applied voltage on resistance wire, the more heat will be transferred to the copper cylinder. The heating temperature will becomes higher, which resulting the mass diffusivity much higher. To interpret this scenario, a close examination of temperature distribution at different applied voltages is shown in Fig. 5. When the applied voltages were $1 \mathrm{v}$ and $2 \mathrm{v}$ respectively, the grayscale intensity images were shown in Fig. 5(a) and 5(b) respectively. Based on the relationship between the fluorescent intensity and temperature obtained from Fig. 3, the color-coded intensity image was shown in Fig. 5(c) and 5(d) respectively. From this figure, we can see that as the voltage applied on the resistance wire increased from $1 \mathrm{v}$ to $2 \mathrm{v}$, the heating temperature increases from $30^{\circ} \mathrm{C}$ to $40^{\circ} \mathrm{C}$, which confirms our above analysis.

\subsection{Effect of flow rate}

The typical images taken in the experiments under the effect of flow rate are shown in Fig. 6, where the applied voltage is kept at $2 \mathrm{v}$. When the flow rate is equal to $1 \mathrm{ml} / \mathrm{h}$, mixing enhancement can be observed as discussed in section 4.1. As the flow rate 
increases from $1 \mathrm{ml} / \mathrm{h}$ to $4 \mathrm{ml} / \mathrm{h}$, the mixing behavior between two fluids in the present microfluidic structure becomes poorer (indicated by the increasingly dark region in the ROI). This can be expected due to that as the flow rate increases, the two fluids flow over the cylinder will have less residence time to be heated. The fluid temperature around the heater will inherently decrease. In order to confirm our analysis, a close examination of fluid temperature field around the heater under the effect of flow rate is shown in Fig. 7. From Fig. 7, we can see that as the flow rate increases from $2 \mathrm{ml} / \mathrm{h}$ to $4 \mathrm{ml} / \mathrm{h}$, the temperature around the heater will decrease.

\subsection{Compare numerical simulation results with experimentalresults}

The typical images taken from the numerical simulation results under the effect of applied voltage are shown in Fig. 8, where the flow rate is kept at $1 \mathrm{ml} / \mathrm{h}$. The concentration field under the effect of applied voltage is shown in Fig. 8 (a) and (b), the corresponding temperature field is shown in Fig. 8 (c) and (d) respectively. From Fig. 8, we can see that as the heating temperature increases, the concentration distribution at the ROI will become more uniform which indicate mixing enhancement. The typical images taken in the experiments under the effect of flow rate are shown in Fig. 9, where the applied voltage is kept at $2 \mathrm{v}$. The concentration field under the effect of flow rate is shown in Fig. 9 (a) and (b), the corresponding temperature field is shown in Fig. 9 (c) and (d) respectively. From Fig. 9, we can see that as the flow rate increases, the temperature around the heater will be decreased, the mixing will inherently becomes poor. Fig. 10(a) shows a comparison between the 
numerical simulations and the experimental results of the mixing efficiency under the effect of applied voltage, which shows that the mixing efficiency increases with increasing applied voltage. Since the thermal chaotic mixing can enhance mixing efficiency, the limiting value is observed to be 0.4 as shown in Fig. 10(a). Fig. 10(b) shows a comparison between the numerical simulations and the experimental results of the mixing efficiency under the effect of flow rate, which shows that the mixing efficiency decreases with increasing flow rate.

\section{Conclusion}

This paper reports a study of thermal chaotic mixing in a T-shaped microchannel with a copper cylinder embedded at the T-junction. Fluorescent dye Acid Yellow and Rhodamine B was employed to show the mass mixing behavior and the temperature field respectively. The effect of power input and flow rate was experimentally investigated. Experimental results show that higher applied voltage, lower flow rate can improve the mass mixing behavior. Mass mixing efficiency was found to be enhanced compared with the mass mixing without heat transfer. However, the heating effect was found to be limited for improving mixing efficiency. The proposed numerical model could give reasonable agreement with the experiment data, which indicates the present numerical model can give a better understanding of the thermal mixing process. 


\section{References}

1. Stroock, A.D., et al., Chaotic mixer for microchannels. Science, 2002. 295(5555): p. 647-651.

2. Nguyen, N.T. and Z. Wu, Micromixers - A review. Journal of Micromechanics and Microengineering, 2005. 15(2): p. R1-R16.

3. Raynal, F. and J.N. Gence, Energy saving in chaotic laminar mixing. International Journal of Heat and Mass Transfer, 1997. 40(14): p. 3267-3273.

4. El Omari, K. and Y. Le Guer, Alternate rotating walls for thermal chaotic mixing. International Journal of Heat and Mass Transfer, 2010. 53(1-3): p. 123-134.

5. Liu, W. and G. Haller, Strange eigenmodes and decay of variance in the mixing of diffusive tracers. Physica D: Nonlinear Phenomena, 2004. 188(1-2): p. 1-39.

6. Popovych, O.V., A. Pikovsky, and B. Eckhardt, Abnormal mixing of passive scalars in chaotic flows. Physical Review E - Statistical, Nonlinear, and Soft Matter Physics, 2007. 75(3).

7. Mokrani, A., C. Castelain, and H. Peerhossaini, The effects of chaotic advection on heat transfer. International Journal of Heat and Mass Transfer, 1997. 40(13): p. 3089-3104.

8. Chagny, C., C. Castelain, and H. Peerhossaini, Chaotic heat transfer for heat exchanger design and comparison with a regular regime for a large range of Reynolds numbers. Applied Thermal Engineering, 2000. 20(17): p. 1615-1648.

9. Acharya, N., M. Sen, and H.C. Chang, Analysis of heat transfer enhancement in coiled-tube heat exchangers. International Journal of Heat and Mass Transfer, 2001. 44(17): p. 3189-3199.

10. Lemenand, T. and H. Peerhossaini, A thermal model for prediction of the Nusselt number in a pipe with chaotic flow. Applied Thermal Engineering, 2002. 22(15): p. 1717-1730.

11. Lefevre, A., et al., Chaotic advection and heat transfer enhancement in Stokes flows. International Journal of Heat and Fluid Flow, 2003. 24(3): p. 310-321.

12. Kumar, V. and K.D.P. Nigam, Laminar convective heat transfer in chaotic configuration. International Journal of Heat and Mass Transfer, 2007. 50(13-14): p. 2469-2479.

13. Yamagishi, A., T. Inaba, and Y. Yamaguchi, Chaotic analysis of mixing enhancement in steady laminar flows through multiple pipe bends. International Journal of Heat and Mass Transfer, 2007. 50(7-8): p. 1238-1247.

14. Lester, D.R., M. Rudman, and G. Metcalfe, Low Reynolds number scalar transport enhancement in viscous and non-Newtonian fluids. International Journal of Heat and Mass Transfer, 2009. 52(3-4): p. 655-664.

15. Sun, Y., Y.C. Kwok, and N.T. Nguyen, Low-pressure, high-temperature thermal bonding of polymeric microfluidic devices and their applications for electrophoretic separation. Journal 
of Micromechanics and Microengineering, 2006. 16(8): p. 1681-1688.

16. Wu, Z., N.T. Nguyen, and X. Huang, Nonlinear diffusive mixing in microchannels: Theory and experiments. Journal of Micromechanics and Microengineering, 2004. 14(4): p. 604-611.

17. Ross, D., M. Gaitan, and L.E. Locascio, Temperature Measurement in Microfluidic Systems Using a Temperature-Dependent Fluorescent Dye. Analytical Chemistry, 2001. 73(17): p. 4117-4123.

18. Incropera, F.P., Liquid Cooling of Electronic Devices by Single-Phase Convection. 1999: John Wiley \& Sons, Inc.

19. R. Weast, M.J.A., W. H. Beyer, CRC handbook of Chemistry and Physics. 1986, Boca Raton: CRC Press Inc.

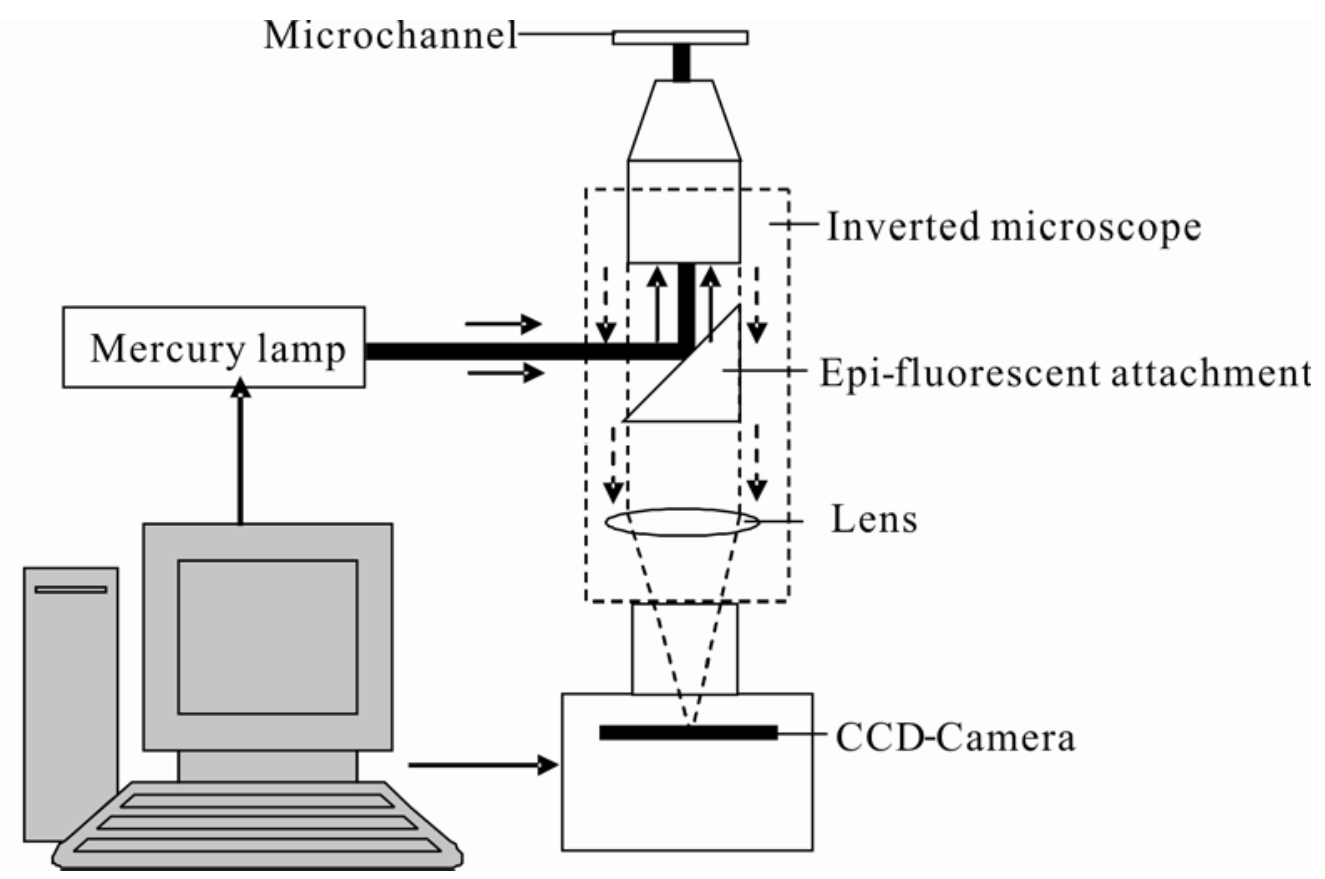

Fig. 1. Schematic illustration of the experimental setup for fluorescence imaging 

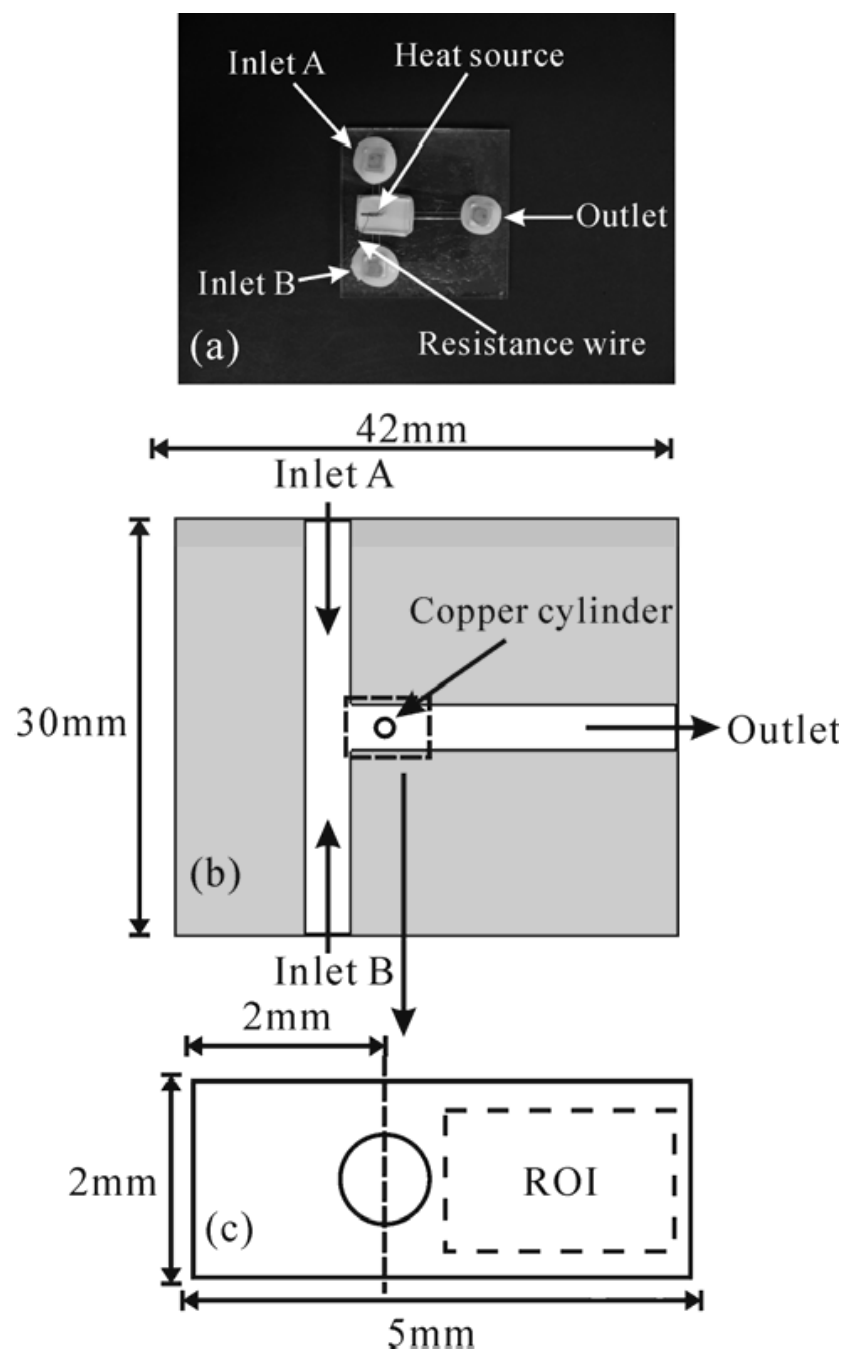

Fig. 2. Schematic illustration of the experimental setup for fluorescence imaging. (a) The

fabricated device used in the experiment, (b) Computational domain, (c) Schematic illustration of focused area captured by CCD camera 


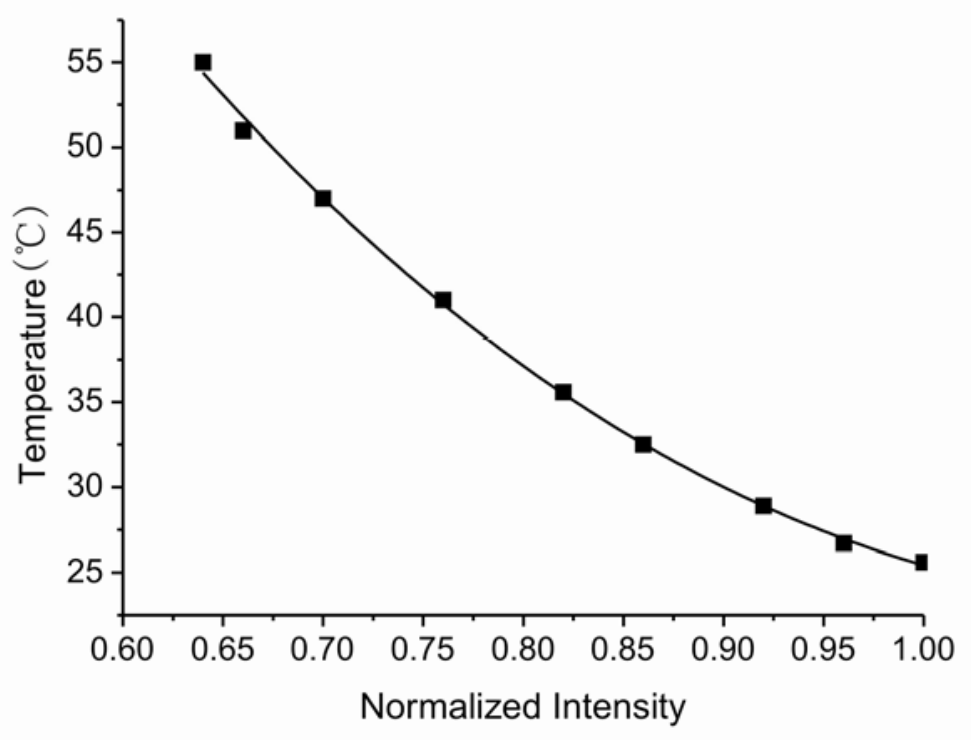

Fig.3. Normalized fluorescence intensity as a function of temperature
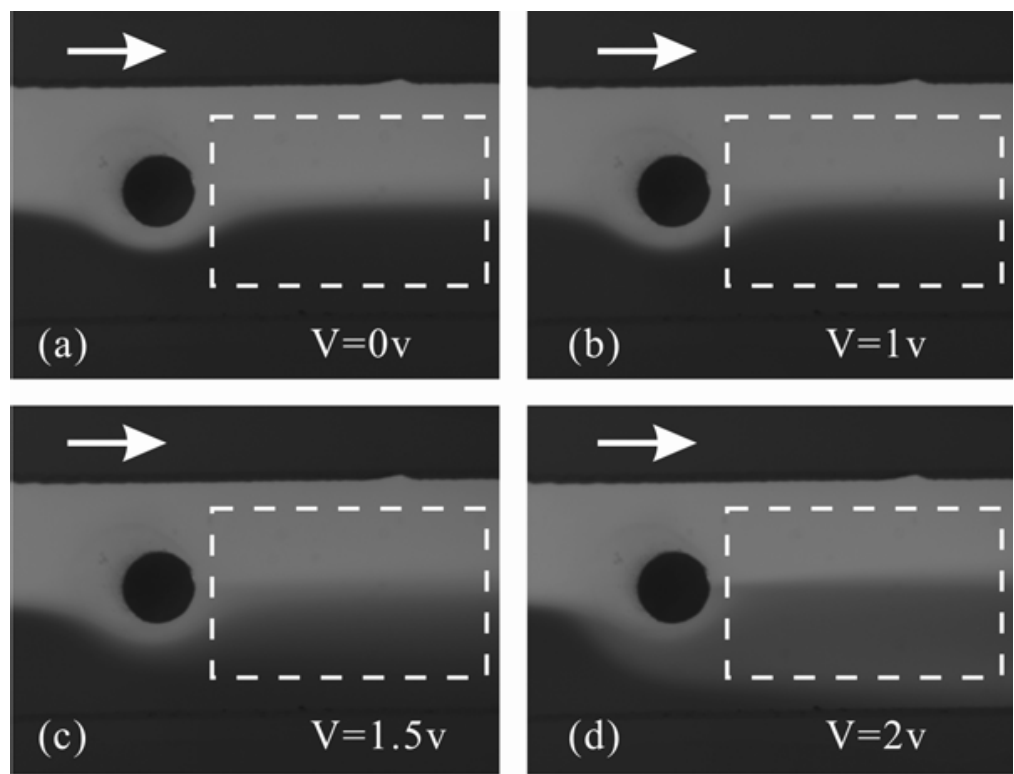

Fig. 4. Thermal chaotic mixing behavior at the around the cylinder. (a) $V=0 v$, (b) $V=1 v$, (c) $V=1.5 v$,

(d) V=2v. The white arrow and box denote the flow direction and ROI respectively. 

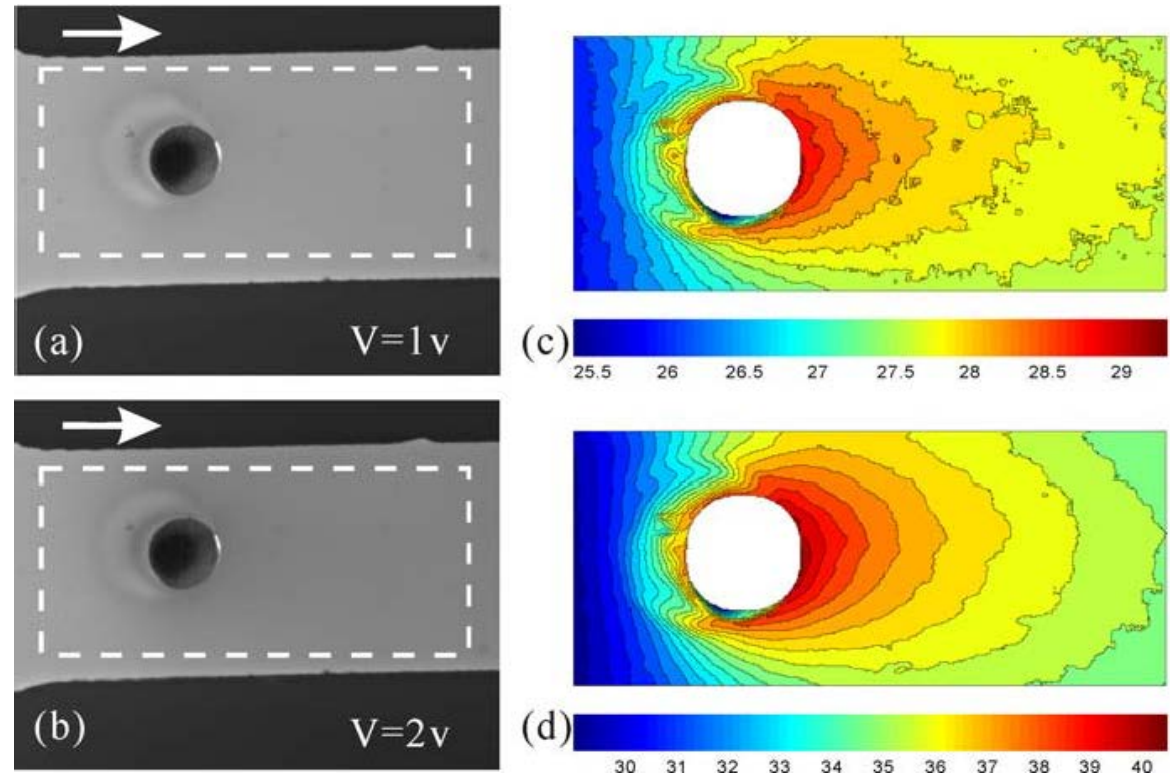

Fig. 5. Measured temperature distribution under the effect of applied voltage (a) original image for $V=1 v$, (b) original image for $V=2 v$, (c) color-coded intensity image for $V=1 v$, (d) color-coded intensity image for $V=2 v$
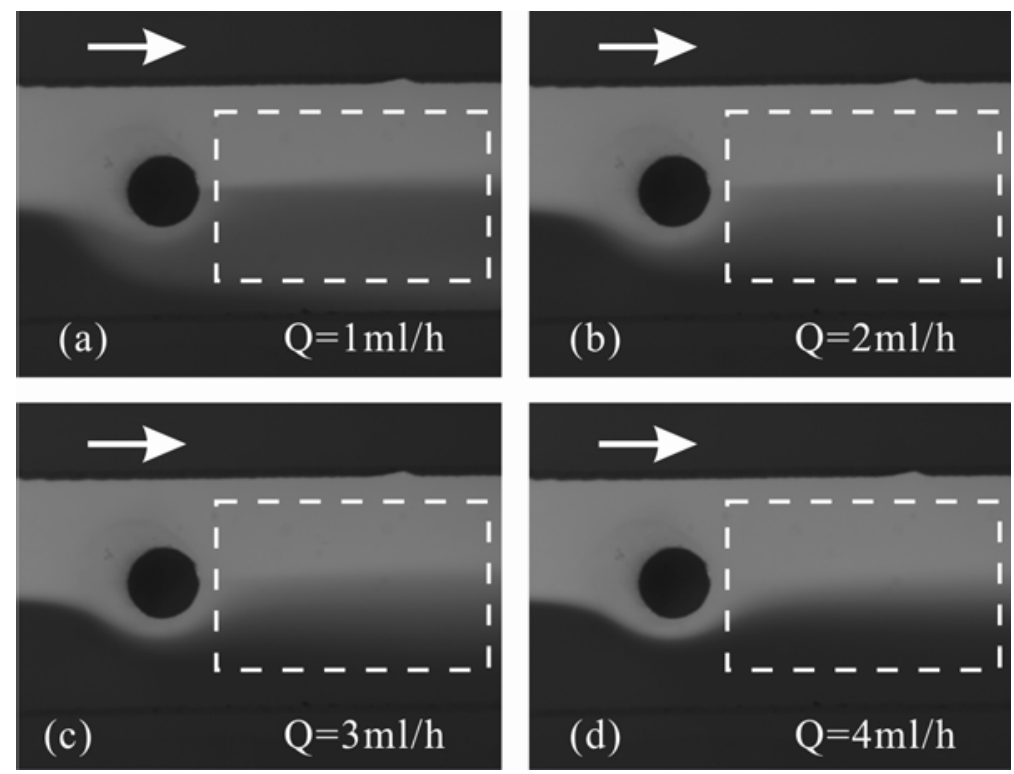

Fig. 6. Thermal chaotic mixing behavior at the around the cylinder. (a) $Q=1 \mathrm{ml} / \mathrm{h},(\mathrm{b}) \mathrm{Q}=2 \mathrm{ml} / \mathrm{h}$, (c)

$\mathrm{Q}=3 \mathrm{ml} / \mathrm{h}$, (d) $\mathrm{Q}=4 \mathrm{ml} / \mathrm{h}$. The white arrow and box denote the flow direction and $\mathrm{ROI}$ respectively. 

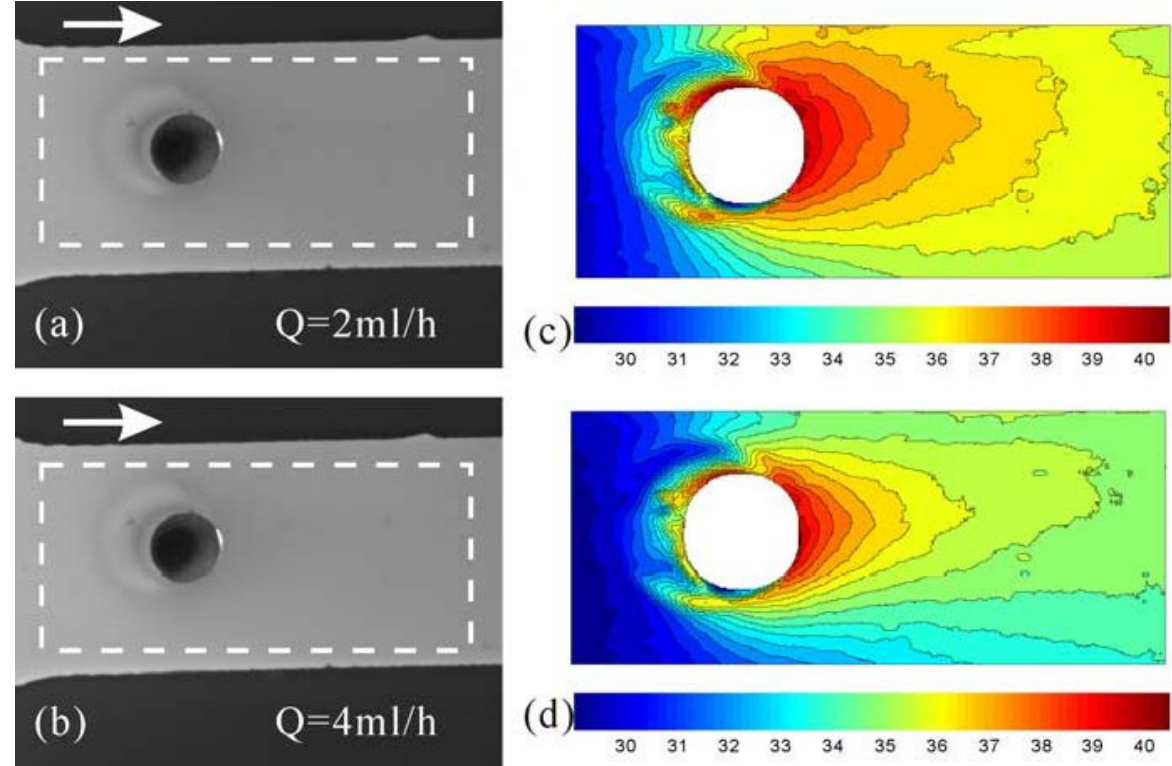

(c)
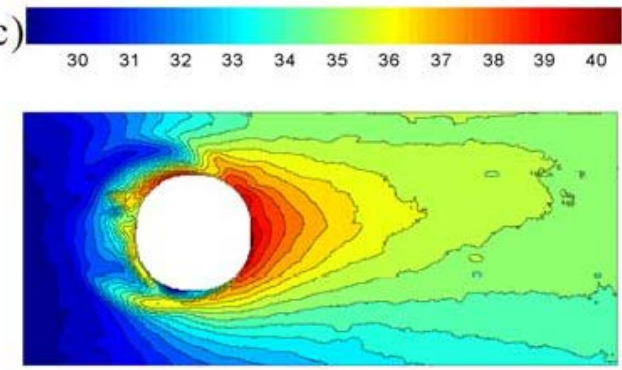

(d)

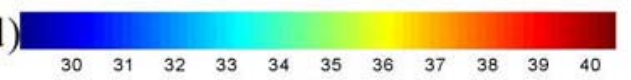

Fig. 7. Measured temperature distribution under the effect of flow rate (a) original image for

$Q=2 \mathrm{ml} / \mathrm{h}$, (b) original image for $Q=4 \mathrm{ml} / \mathrm{h}$, (c) color-coded intensity image for $Q=2 \mathrm{ml} / \mathrm{h}$, (d)

color-coded intensity image for $Q=4 \mathrm{ml} / \mathrm{h}$
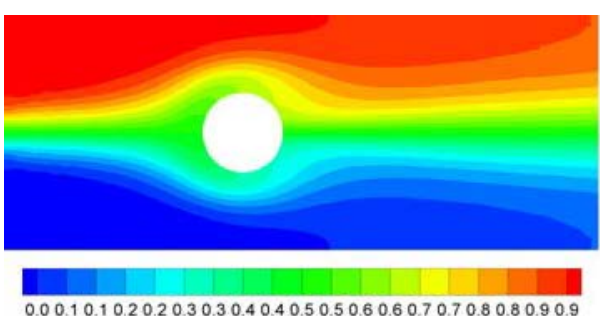

(a)

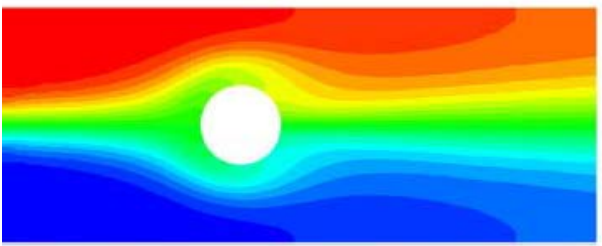

0.00 .10 .10 .20 .20 .30 .30 .40 .40 .50 .50 .60 .60 .70 .70 .80 .80 .90 .9

(b)

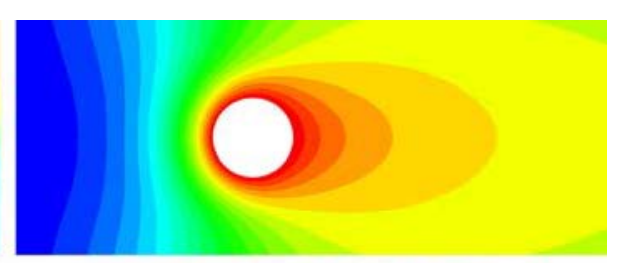

26262727272727282828282829292929293030

(c)

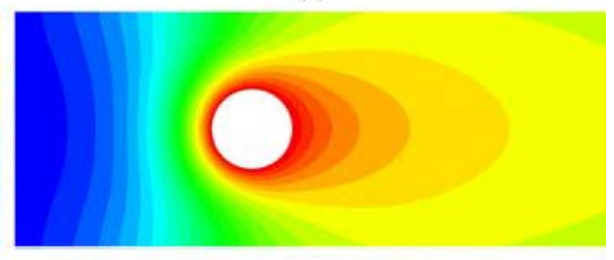

2829293030313132323333343435353636373738383939

(d)

Fig. 8. Numerical simulation result of concentration and temperature field under the effect of

applied voltage (a) concentration field for $V=1 v$, (b) concentration field for $V=2 v$, (c) temperature

field for $V=1 v$, (d) temperature field for $V=2 v$ 


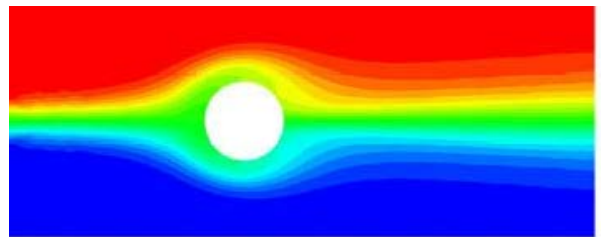

0.00 .10 .10 .20 .20 .30 .30 .40 .40 .50 .50 .60 .60 .70 .70 .80 .80 .90 .9

(a)

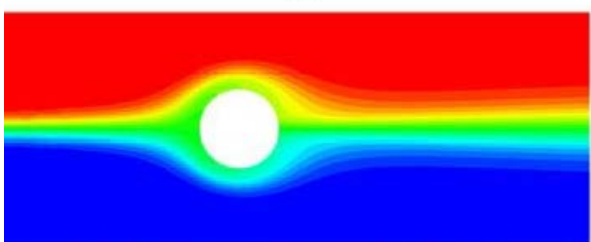

0.00 .10 .10 .20 .20 .30 .30 .40 .40 .50 .50 .60 .60 .70 .70 .80 .80 .90 .9

(b)

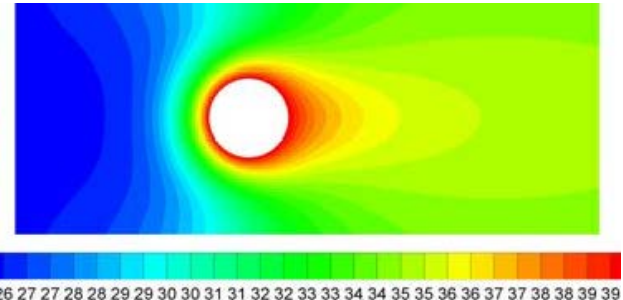

(c)

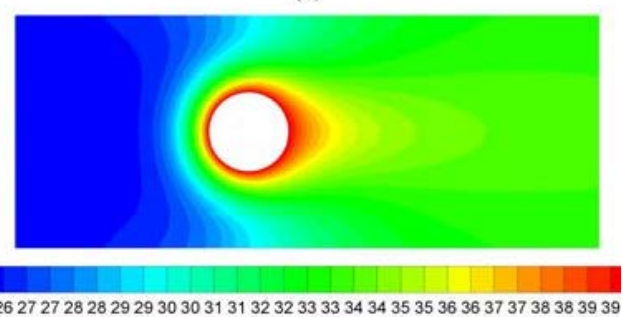

(d)

Fig. 9. Numerical simulation result of concentration and temperature field under the effect of

flow rate (a) concentration field for $Q=2 \mathrm{ml} / \mathrm{h}$, (b) concentration field for $Q=4 \mathrm{ml} / \mathrm{h}$, (c)

temperature field for $Q=2 \mathrm{ml} / \mathrm{h}$, (d) temperature field for $Q=4 \mathrm{ml} / \mathrm{h}$

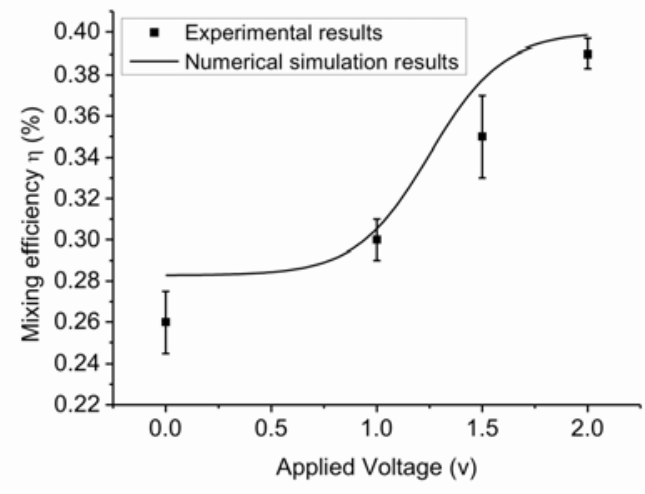

(a)

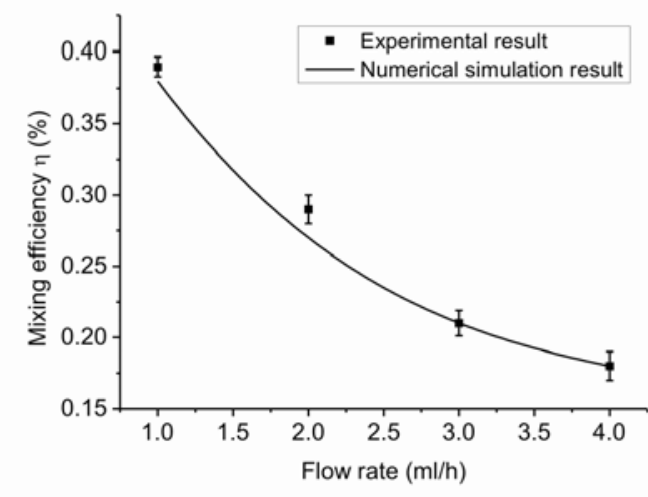

(b)

Fig. 10. Comparison between numerical simulation results and experimental results of the mixing

efficiency (a) under the effect of applied voltage, (b) under the effect of flow rate 